AUTHOR(S): $\quad$ K(laus) S. Lackner, T-3

S(tirling) A. Colgate, T-6

N(orman) L. Johnson, T-3

R(onald) C. Kirkpatrick, X-1

R(alph) Menikoff, T-14

A(lbert) G. Petschek, P-DO/AFF

SUBMITTED TO:

11 th International Workshop on Laser Interaction and Related

Plasma Phenomena, Monterey, California, October 25-29, 1993 
This report was prepared as an account of work sponsored by an agency of the United States Government. Neither the United States Government nor any agency thereof, nor any of their employees, makes any warranty, express or implied, or assumes any legal liability or responsibility for the accuracy, completeness, or usefulness of any information, apparatus, product, or process disclosed, or represents that its use would not infringe privately owned rights. Reference herein to any specific commercial product, process, or service by trade name, trademark, manufacturer, or otherwise does not necessarily constitute or imply its endorsement, recommendation, or favoring by the United States Government or any agency thereof. The views and opinions of authors expressed herein do not necessarily state or reflect those of the United States Government or any agency thereof.

\title{
EQUILIBRIUM IGNITION FOR ICF CAPSULES
}

\author{
K.S. Lackner, S.A. Colgate. N. L. Johnson. \\ R.C. Kirkpatrick. R. Menikoff \& A. G. Petschek \\ Los Alamos National Laboratory
}

\begin{abstract}
In comparing high-gain ICF targets using cryogenic DT for a pusher with equilibrium ifsnition targets using high- $Z$ pushers which contain the radiation. we point to the intrinsic advantages of the latter. Equilibrium or volume ignition sacrifices high gain for lower losses. lower ignition temperature, lower implosion velocity and lower sensitivity of the more robust. capsule to small fluctuations and asymmetries in the drive system. The reduction in gain is about a factor of 2.5. which is small enough to make the more robust equilibrium ignition an attractive alternative.
\end{abstract}

\section{INTRODUCTION}

There are two fundamentally different approaches to igniting DT fuel in an ICF capsule which can be described as equilibrium and hot spot ignition. In both cases. a capsule which can be thought of as a pusher containing the DT fuel is imploded until the fuel reaches ignition conditions. High gain. hot spot ignition uses cold DT as a pusher. Both the pusher and the fuel are transparent and ignition occurs out of thermal equilibrium with significant radiation losses. The advantage is that the surrounding. cold DT can also burn and at least in principle lead to high gain. In contrast. equilibrium ignition uses a heavy metal pusher to confine the hot DT as well as the radiation. The radiation smoothes out any initial temperature variations in the fuel. This approach sacrifices the potential for high gain for the advantages of lower losses, a lower ignition temperature. a lower implosion velocity and a lower sensitivity to instabilities in the implosion system.

The ICF program has largely concentrated on high gain. hot spot ignition and only a few researches have emphasized the advantages of equilibrium ignition or volume burn. see for example Refs. 1-6. It is the purpose of this paper to compare equilibrium burn to hot spot ignition and show that equilibrium ignition presents a viable alternative that deserves a more thorough analysis. Unfortunately, it is not possible to simply extrapolate from the experience with hot spot ICF to equilibrium approaches since the two approaches are subject to rather different constraints and limitations.

\section{THE DT FUEL}

In this section we outline the dynamics of the fuel as it approaches ignition. For both systems, the most easily ignited fuel is DT and we will restrict our discussion to that. In high gain, hot spot ignition the pusher itself is made from cryogenic DT which 
ultimately is burned. too. For purposes of this discussion we consider the ablator shell on the outside of the pusher as part of the driver rather than of the capsule. In the case of equilibrium ignition, the pusher is made from a high- $Z$. dense material like gold which can contain the radiation and requires significantly lower specific energy to reach a given pressure than lighter materials.

The imploding pusher compresses the fuel until it reaches ignition. By this we mean the point at which fusion reactions add so much heat that they can support and maintain themselves. (For a more refined definition and discussion of ignition see Ref. 7.) Adding a little more energy to a capsule that barely reaches ignition causes the fusion reaction to run away, i.e. to consume a significant fraction of the fuel before disassembly.

For the fusion reaction to bootstrap itself, a significant amount of the fusion energy must be deposited into the fuel. This requires a fuel thickness at turnaround sufficient to stop the $\alpha$-particles produced in the reaction. Their stopping distance, measured in $\rho R$, depends on the temperature $T$,

$$
\langle\rho R\rangle_{\mathrm{ign}} \approx 5.2 \times 10^{-3}\left(T_{\mathrm{ign}} /[\mathrm{keV}]\right)^{3 / 2} \mathrm{~g} / \mathrm{cm}^{2}
$$

The conditions under which the fuel ignites are rather different in the two cases. In hot spot ignition the pusher cannot contain the radiation from the hot fuel. Thus. the fusion rate has to overcome radiation losses. before ignition is reached. These losses are due to electron bremsstrahlung which is proportional to

$$
\left(n_{D}+n_{T}+\sum_{i} Z_{i} n_{i}\right)\left(n_{D}+n_{T}+\sum_{i} Z_{i}^{2} n_{i}\right) T^{1 / 2} .
$$

$n_{i}$ and $Z_{i}$ are the number density and ionization state respectively. The sum extends over the impurity ions in the mixture.

For pure DT, the fusion rate overcomes bremsstrahlung losses at about $5 \mathrm{keV}$ independent of the fuel density (ideal ignition temperature ${ }^{8,9}$ ) At this temperature $\langle\rho R\rangle_{\text {ign }}$ is small. In practice, there are other losses and an ignition temperature of $10 \mathrm{keV}$ and $\langle\rho R\rangle_{\text {ign }}=0.3 \mathrm{~g} / \mathrm{cm}^{2}$ are considered typical. ${ }^{9-11}$

In contrast, in equilibrium ignition, the high opacity of the gold prevents radiation from leaving the fuel volume. As a result the radiation field will rapidly reach its equilibrium black body spectrum. The system will sustain a certain heat loss into the wall. which is best described as a Marshak wave traveling into the wall. ${ }^{4}$ Fortunately, at the temperatures and pressures envisioned this only involves a very small fraction of the wall. In effect it will act like an increased heat capacity of the fuel. In a first approximation one is justified in ignoring this loss rate. Thus the hydrodynamic compression only has to heat the gas to a temperature at which heat deposition from fusion in the stopping time ( $\sim$ minimum $\mathrm{R}$ /implosion speed) adds a comparable amount of energy. This assures that fusion reactions can bootstrap themselves causing a significant energy release in the expansion phase. Roughly, ignition occurs at $1.5 \mathrm{keV}$ and losses will raise it to about $2 \mathrm{keV}$. At such a low temperature, the $\langle\rho R\rangle_{\text {ign }}$ required for stopping $\alpha$-particles is very small, $0.016 \mathrm{~g} / \mathrm{cm}^{2}$. This would open the possibility for very small capsule experiments near the ignition point, if it were not for heat losses to the wall. These losses can be reduced by increasing $\langle\rho R\rangle$ and suggest $\langle\rho R\rangle_{\mathrm{ign}}=0.3 \mathrm{~g} / \mathrm{cm}^{2}$ which is also necessary to sustain burn. ${ }^{4}$ 
Radiation smoothes out variations inside an equilibrium capsule while leading to a steep temperature gradient in a surface layer of the pusher. Immediately prior to ignition. radiation and hydrodynamics will smooth the temperature and pressure of the fuel which will be approximately uniform in $T . P$ and $\rho$. Approaching ignition. the entire fuel volume is in thermal equilibrium between electrons. ions and radiation. After ignition, of course, energy deposition happens too rapidly to maintain thermal equilibrium. For a detailed discussion see Ref. 2.

In hot spot ignition it is, however, possible to maintain a variation in the entropy of the fuel. Of course, near ignition the $\alpha$-deposition will evenly heat a volume of $0.3 \mathrm{~g} / \mathrm{cm}^{3}$, thus leading to a hot spot volume that is quite comparable to the minimum fuel volume in equilibrium ignition. In spite of their suggestive names. in terms of the required $\langle\rho R\rangle$ the two systems are rather similar. Ignition temperatures, however. differ by a factor of five.

The two different approaches to ICF differ in their sensitivity to mix. In tokamak fusion one major difficulty has been the elimination of high- $Z$ impurities. ${ }^{12}$ and one can expect this also to become a significant issue for hot spot ignition where the ablator is a likely source of impurities. Since bremsstrahlung is proportional to $\langle Z\rangle\left\langle Z^{2}\right\rangle$. the increase in radiation due to impurities dramatically increases the ignition temperature. Even a five percent admixture of silicon atoms would completely prevent ignition. Radiation losses from only partially ionized high- $Z$ impurities is even larger due to line radiation which can exceed bremsstrahlung and further increases the sensitivity of the ignition temperature to impurities. As a result instabilities injecting high- $Z$ ablator material into the outer layer of the pusher wonld significantly reduce gain. Injection into the hot spot could effectively quench the fusion reaction. Even mixing pure DT into the hot spot is deleterious if it occurs in chunks. At constant pressure the radiation rate per unit volume is proportional to $T^{-3 / 2}$. Hence, a mixture of cold and hot DT at constant pressure radiates more strongly than a homogeneous mixture.

The major effect of mix on equilibrium ignition capsules is to increase the heat capacity of the fuel and thus lower its temperature. As long as the added mass is small compared to the fuel such an effect is tolerable. Because of the higher heat capacity of high- $Z$ materials it may even be advantageous to add a small amount of xenon to the fuel. This would increase the work required in heating the fuel but would make it insensitive to late stage admixtures of cool pusher material.

\section{THE PUSHER}

Critical for the design of a pusher is that at turnaround it must contain the fucl pressure which implies that it reaches similar pressures. To gain a qualitative understanding of the dynamics. it is useful to make the simplifying assumption that the entire pusher is brought to rest at turnaround. The approximate validity of this assumption can be checked with numerical calculations.

We assume that the drive system can bring the pusher to an implosion velocity $v_{0}$. At turnaround its kinetic energy will be transformed into internal energy plus the energy of the fuel. As noted above, the fuel can be characterized by $\langle\rho R\rangle_{\text {ign }}$ and $T_{\mathrm{ign}}$. Thus. we consider, in addition to $v_{0} .\langle\rho R\rangle_{\mathrm{ign}}, T_{\mathrm{ign}}$, and $P_{\mathrm{ign}}$ as the independent variables describing the capsule. Of these. only $P_{\mathrm{ign}}$ is still undetermined. Written in these variables the fuel mass becomes

$$
m_{f}=\frac{4 \pi}{3}\langle\rho R\rangle_{\mathrm{ign}}^{3}\left[(\gamma-1) C_{f} T_{\mathrm{ign}} / P_{\mathrm{ign}}\right]^{2} .
$$


with $\gamma \sim 5 / 3$ and $C_{f}=1.16 \times 10^{3}\left(\mathrm{Mbcm}^{3} / \mathrm{g}\right) / \mathrm{keV}$. In the absence of losses. energy conservation implies

$$
M v_{0}^{2} / 2=M E_{p}\left(P_{\mathrm{ign}}\right)+m_{f}\left(\langle\rho R\rangle_{\mathrm{ign}}, T_{\mathrm{ign}}, P_{\mathrm{ign}}\right) C_{f} T_{\mathrm{ign}}
$$

where $M$ is the pusher mass and $E_{p}$ the specific internal energy of the pusher at turnaround.

One can now vary $P_{\text {ign }}$ until $M$ and thus the total energy $\frac{1}{2} M v_{0}^{2}$ is minimized. $d M / d P_{\text {ign }}=0$ implies

$$
\frac{d E_{p}}{d P_{\mathrm{ign}}}=-\left[\frac{1}{m_{f}} \frac{d m_{f}}{d P_{\mathrm{ign}}}\right] \cdot\left[\frac{v_{0}^{2}}{2}-E_{p}\left(P_{\mathrm{ign}}\right)\right]
$$

$d m_{f} / d P_{\text {ign }}=-2 m_{f} / P_{\text {ign }} . \quad d E / d P_{\text {ign }}$ only depends on the equation of state of the pusher. For heavy metals at high pressure $E \approx E_{\text {ref }}\left(P / P_{\text {ref }}\right)^{q}$. leading to

$$
E_{p}=\frac{v_{0}^{2} / 2}{1+q / 2}
$$

For an optimum target. the specific internal energy of the pusher at turnaround only depends on the pusher EOS and the implosion velocity. This implies that the optimal energy ratio between fuel and pusher is fixed and independent of $T_{\mathrm{ign}}$ and $\langle\rho R\rangle_{\mathrm{ign}}$. With $q$ empirically on the order of 0.5 , about $80 \%$ of the initial energy remains in the pusher in order to provide a high ignition pressure.

In comparison with simple numerical calculations we have performed, we find these estimates quite reasonable for equilibrium ignition capsules. However, in the case of hot spot ignition they are far too optimistic because they ignore radiative losses.

Even from such a simple model one can obtain a number of important insights:

(1) For an optimal design, a large fraction of the energy invested remains in the pusher.

(2) The maximum achievable pressure determines the minimum mass of the fuel. Lower pressures at ignition temperature imply a lower density and thus require a larger radius and an increase in mass to maintain $\rho R$.

(3) The ignition pressure is proportional to $v_{0}^{2 / 4}$. Thus the characteristics of the drive system which tend to limit the achievable $v_{0}$ greatly affect the ignition pressure and the minimum energy requirement. The minimum energy is a steep function of the implosion velocity.

(4) Since high- $Z$ materials have smaller values of $E_{\text {ref }} / P_{\text {ref }}^{q}$, for given $v_{0}$ the turnaround pressure grows with the pusher density. Thus, there is an intrinsic advantage in high density pushers. In practice, transparent low density pushers obtain similar energy densities by using much higher implosion velocities. However, they also have to compensate for radiative losses and the higher ignition temperature.

An additional advantage of the high- $Z$ pusher is that the energy content of the pusher is little affected by the drive history. Because the pressure in the pusher at turnaround must match that of the fuel, additional energy is required if the pusher is set on a higher adiabat. For our estimates of the high density, heavy metal pusher we typically assume that the adiabat is set by an initial shock traversing the pusher at 
Table 1: Comparison of Idealized Equilibrium Capsule and Hot Spot Capsule Designs.

\begin{tabular}{lccl} 
& equilibrium & hot spot \\
\hline velocity & 10 & 40 & $\mathrm{~cm} / \mu \mathrm{s}$ \\
ignition temperature & 2 & 10 & $\mathrm{keV}$ \\
fuel mass & 0.2 & 0.1 & $\mathrm{mg}$ \\
pusher mass & 40 & 5 & $\mathrm{mg}$ \\
total energy & 0.2 & 0.4 & $\mathrm{MJ}$ \\
initial radius & 1.3 & 2.8 & $\mathrm{~mm}$ \\
drive pressure & 200 & 43 & $\mathrm{Mb}$ \\
initial aspect ratio & 15 & 10 & \\
convergence ratio & $10-20$ & $20-30$ & \\
Burn fraction & 0.6 & 0.1 & \\
maximum gain & 180 & 440 & \\
\hline
\end{tabular}

* Excluding pusher DT

$100 \mathrm{Mb}$. It turns out that even such a strong shock does not greatly change the energy requirement. The DT pusher on the other hand is affected strongly for two reasons: a DT pusher requires significantly more energy if shocked even to as low a value as $10 \mathrm{Mb}$; on such an adiabat the DT pusher would loose a large amount of energy to radiation. Since the radiation is proportional to $M P T^{-1 / 2}$, the radiative losses from the capsule would be dominated by the pusher which contains most of the mass. Only by keeping the pusher electrons degenerate. can these losses be reduced. ${ }^{13} \mathrm{~A}$ careful shaping of the pressure pulse driving the DT capsule is required in order to maintain a cold pusher while at the same time setting a high adiabat for the $\mathrm{D}^{\prime} \mathrm{T}$ in the hot spot. Achieving the high adiabat and compressing the fuel to the necessary hot spot pressure also requires a high implosion symmetry. Equilibrium burn is much less sensitive to pulse shape; the drive pressure history requires little tailoring and even an asymmetric convergence can still yield high pressures and temperatures in the fuel.

\section{GAIN FACTORS}

The gain that can be obtained in the two designs depends critically on the burn fractions. Because of the robustness and the large containment time, we believe that high burn fractions are possible in high- $Z$, equilibrium capsules. Note again, that mixing and radiation losses in the late stages are not likely to quench the system. We consider burn fractions exceeding $60 \%$ as achievable. In contrast, hot spot ignition is subject to quenching, particularly in the outer region of the pusher, which is at a low density, is not well confined by inertia, and furthermore is subject to impurity admixtures from the ablator. Therefore we consider a $10 \%$ burn fraction to be a realistic estimate.

With these assumptions, one can estimate the gain factor of the capsule-fuel system in the two cases. Losses due to instabilities are ignored, but unavoidable heat losses are accounted for by assuming a nominally high value of the ignition temperature. In the case of hot spot ignition with an implosion velocity of $40 \mathrm{~cm} / \mu$ s the average energy investment is $800 \mathrm{Mbar} \mathrm{cm}^{3} / \mathrm{g}$ or $40 \mathrm{keV}$ per fusion reaction. This simple estimate leads to a gain factor of about 440 . 
In the case of the high- $Z$ pusher the temperature in the fuel is $2 \mathrm{keV}$. This corresponds to $12 \mathrm{keV}$ per DT. The total energy investment into the pusher and the fuel is five times larger. At a burn fraction of $60 \%$ one invests $100 \mathrm{keV}$ per fusion reaction leading to a gain that is only a factor of 2.5 lower than for hot spot ignition. Our result obtained from this very simple estimate is in good agreement with the analytical and numerical work by Basko. ${ }^{5}$

\section{CONCLUSIONS}

Estimates for idealized capsules lead to the comparison summarized in Table 1. Most of the data involving hot spot ignition are taken from Refs. 11 and 10. In the case of the equilibrium capsule, the effects of losses have at least in part been accounted for by a raised ignition temperature and the choice of $\langle\rho R\rangle$. Similarly, for hot spot ignition losses have been included by chosing a relatively high value for the pusher velocity. We note that the two designs are not that different in their requirements on a drive system. While the equilibrium capsule requires a lower pusher velocity, the average drive pressure must be significantly higher. Because of the lower mass, the nonequilibrium capsule can achieve a significantly higher velocity for the same initial aspect ratio and drive pressure. Overall, maximum achievable gains are not that different.

This suggests that one should seriously reconsider whether the "high gain" of the hot spot ignition is indeed worth the additional complications. Clearly, heavy metal pushers have many advantages concerning robustness and stability and can lead to respectable gain factors.

\section{REFERENCES}

1. H. Hora \& P. S. Ray, "Increased Nuclear Fusion Yields of Intertially Confined DT Plasma due to Reheat," Zeitschrift für Naturforschung 33A (1978), 890-894.

2. R.C. Kirkpatrick \& J.A. Wheeler, "The Physics of DT Ignition in Small Fusion Targets," Nuclear Fusion 21 (1981). 389-401.

3. S. Eliezer, H. Hora, "The Physics of Directly Driven Targets," in Nuclear Fusion by Inertial Confinement. G.Velarde \& Y. Ronen \& J. M. Martínez-Val, eds., CRC Press, Boca Raton, 1993. 44-70.

4. Stirling A. Colgate \& Albert G. Petschek, "Minimum Conditions for the Ignition of Fusion in Thermal Equilibrium with Radiation," Unpublished Los Alamos Report, December 1989.

5. M. M. Basko, "Spark and Volume Ignition of DT and $D_{2}$ Microspheres," Nuclear Fusion 30 (1990). 2443-2451.

6. H. Hora. S. Eliezer, J. M. Martínez-Val \& G. H. Miley, "High Gain Volume Ignition at ICF.” 1993. These Proceedings.

7. R.C. Kirkpatrick, "Ignition critical profiles for small fusion targets." Nuclear Fusion 21 (1081). 1457-1466.

8. Samuel Glasstone \& Ralph H. Lovberg, in Controlled Thermonuclear Reactions, D. Van Nostrand Company, Inc., Princeton, New Jersey, 1960.

9. Richard F. Post, "Controlled Fusion Research-An Application of the Physics of High Temperature Plasmas," Reviews of Modern Physics 28 (1956), 338-362.

10. John D. Lindl. "Physics of Ignition for ICF Capsules," in Varenna Summer School, 1988. 617-630.

11. John D. Lindl, "Introduction to the Physics of ICF Capsules," in Varenna Summer School, 1988. 595-615.

12. M. Keilhacker \& the JET team, "Overview of results from the JET tokamak using a beryllium first wall," Phys. Fluids B 2(1990), 1291-1299.

13. J. Meyer-ter-Vehn, "On Energy Gain of Fusion Targets: The Model of Kidder and Bodner Improved," Nuclear Fusion 22 (1982), 561-565. 

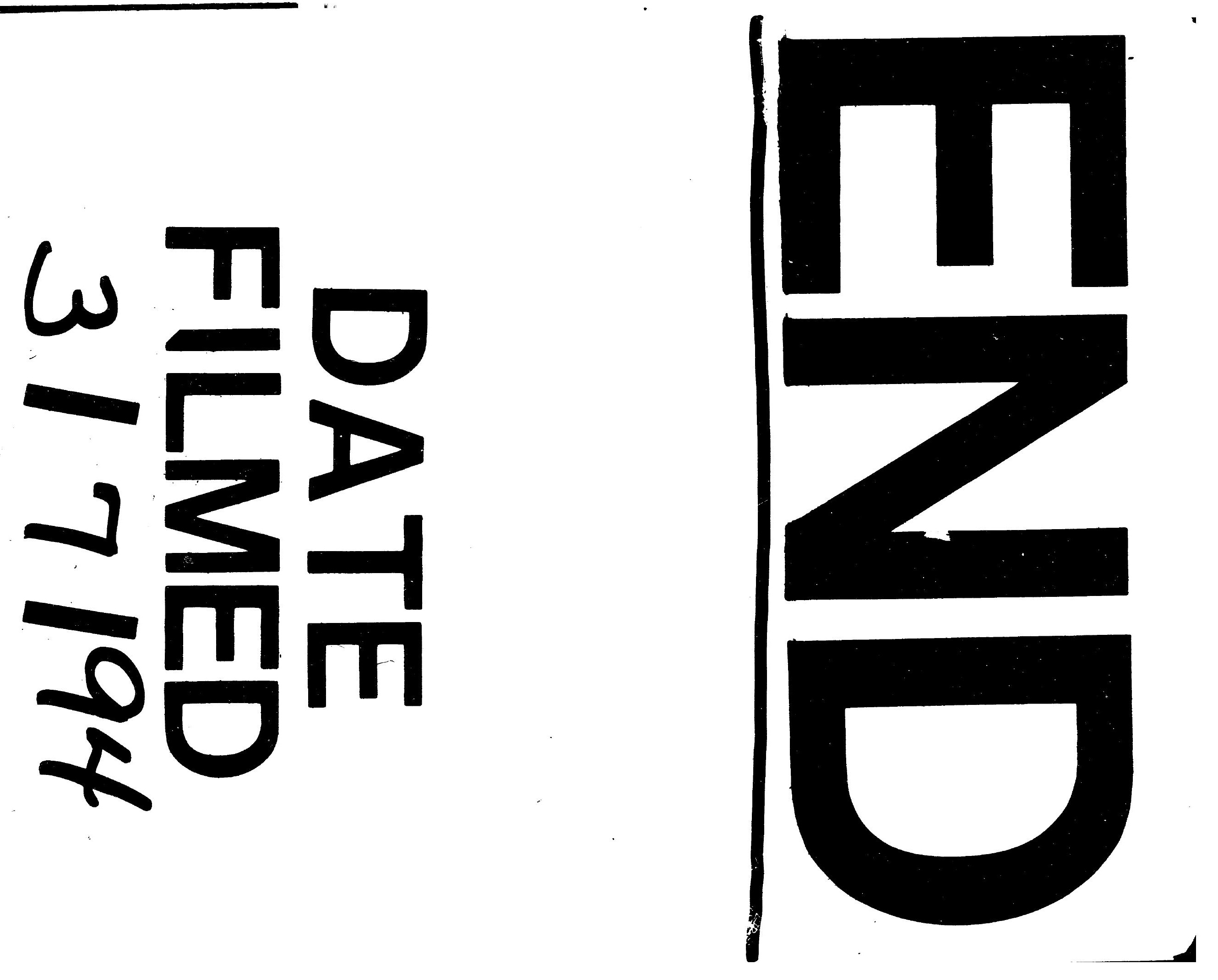
